Communications in Physics, Vol. 28, No. 2 (2018), pp. 139-153

DOI:10.15625/0868-3166/28/2/11040

\title{
WAVELENGTH SHIFT MEASUREMENT METHOD WITHOUT SPECTROMETER: THE NEW WAY FOR ENVIRONMENT PHOTONIC SENSORS
}

\author{
PHAM VAN HOI ${ }^{a, b \dagger}$, NGUYEN THUY VAN ${ }^{a, b}, \mathrm{PHAM} \mathrm{VAN} \mathrm{DAI}^{a}, \mathrm{BUI} \mathrm{HUY}^{a, b}$, \\ LE HUU THANG ${ }^{b, c}$, NGUYEN VAN AN $^{b, d}$ AND PHAM THANH BINH ${ }^{a}$ \\ ${ }^{a}$ Institute of Materials Science, Vietnam Academy of Science and Technology (VAST) \\ ${ }^{b}$ Graduated University of Science and Technology, VAST \\ ${ }^{c}$ Small and Medium Enterprise Development and Support Center 1, STAMEQ, \\ 8 Hoang Quoc Viet, Cau Giay, Hanoi, Vietnam \\ ${ }^{d}$ University of Science, Hue University, 77 Nguyen Hue str., Hue city, Vietnam \\ $\dagger$ E-mail: hoipv@ims.vast.ac.vn \\ Received 2 January 2018 \\ Accepted for publication 6 May 2018 \\ Published 23 June 2018
}

\begin{abstract}
In this paper, we overview some configurations of photonic devices for measuring mechanical strain and wavelength shift without use of spectrometer, which have been developed recently and demonstrated large potential application in sensing technique with low cost. There are three kinds of photonic sensor configurations to be presented: i) first of them is a fiber Bragg grating (FBG) for the measurement of mechanical dynamic strain of very small value using signal modulation on laser diode by composition of two wavelengths in the laser cavity; ii) second configuration is a FBG combined with a DFB laser diode whose emission wavelength controlled by laser temperature, and iii) last one uses a fiber ring laser from Erbium doped fiber and two FBG operated as reference and sensing probe. For the environment control, the etched-fiber Bragg grating (e-FBG) as sensing probe is very suitable for bio- and/or chemical sensors. The photonic sensors can increase sensitivity and measuring accuracy by narrow line-width of reflection laser spectra and, therefore, the sensors can determine a refractive index variation of $2 \times 10^{-4}$, which is similarly as use of a high resolution spectrometer. The experimental results show that this sensing method could determine different mixing ratios of organic solvents in liquid environment with good repeatability, high accuracy and rapid response.
\end{abstract}

Keywords: photonic sensors, fiber Bragg grating, environment control.

Classification numbers: 42.81.Pa, 42.79.Dj, 07.60.Vg.

(C)2018 Vietnam Academy of Science and Technology 


\section{INTRODUCTION}

Optical fiber sensors offer very attractive solutions over conventional technologies due to some unique characteristics such as multiplexing capabilities, high sensitivity, fast response, immunity to electromagnetic interference, and enable for the remote in-situ sensing of species in difficult or hazardous environments [1,2]. Fiber Bragg gratings (FBGs) have been demonstrated as optical sensors for various applications [3], especially for chemical and biochemical sensing [4]. In various chemical and biochemical applications, refractive index sensing method is important, since several substances can be detected by the measurement of refractive indices. The FBG sensing operation principle involves detecting the central wavelength variation. However, conventional spectrum interrogation methods utilizing an optical spectrum analyzer (OSA) with high-resolution is expensive. Therefore, it is of significance to find FBG interrogation methods with high resolution and without use of optical spectrum analyzer. The general requirements for an ideal interrogation method are as follows: high resolution with large measurement range, cost effective and compatible with multiplexing. The demodulation techniques are divided into two categories based on the light source, which is either broadband (e.g., amplified spontaneous emission) or narrowband (e.g., semi-conductor tunable laser source) [5]. The narrowband light source usually has higher sensitivity, which could be improved further with other techniques such as phase-shifted FBG and balanced detection [6].

In this paper, we overview some configurations of FBG- devices for measuring wavelength shift without use of spectrometer, which have potential applications in mechanical displacement measurement and environment sensing technique with low cost. There are three configurations of photonic sensors to be presented: first of them is FBG sensor for measurement of mechanical dynamic strain of very small value using signal modulation on a laser diode by composition of two wavelengths in the laser cavity; second one is a determination of FBG wavelength shift by tuning the lasing wavelength of a DFB single-mode laser due to the change of laser substrate temperature, and the last configuration is a sensing system based on dual FBGs integrated in a fiber ring laser from Erbium-doped fiber and two FBG operated as reference and sensing probe. The etched-fiber Bragg grating (e-FBG) as sensing probe is suitable for bio- and/or chemical sensors. The e-FBG sensor can be used to investigate different types of the organic compounds, gasoline and RON92 added ethanol and/or methanol with concentration range of $0 \%-14 \%$.The distinguishing advantage of this scheme is due to the use of a fiber laser and the signal-noise-ratio (SNR) can be improved. The line-width spectrum of fiber laser emission is much narrower than that of the reflected FBG spectra, thus detecting accuracy and capability of the sensing system can be enhanced for remote sensing.

\section{PRINCIPLE OF FBG-SENSOR SYSTEMS}

\section{II.1. Principle of dynamic strain sensor using FBG}

The Bragg grating wavelength $\left(\lambda_{B}\right)$ is the center wavelength of the light reflected from a Bragg grating, that depends upon the effective index of the fiber core $\left(\mathrm{n}_{e f f}\right)$ and the periodicity of the Bragg grating $(\Lambda)$ through the relation $\lambda_{B}=2 \mathrm{n}_{e f f} \Lambda$. The operating principle of the longitudinal dynamic strain FBG sensor system using optical feedback interferometry is applied from one of basic applications of self-mixing effect that is the measured displacement of the target. The longitudinal dynamic strain is provided when the cantilever-beam is excited by a mechanical 


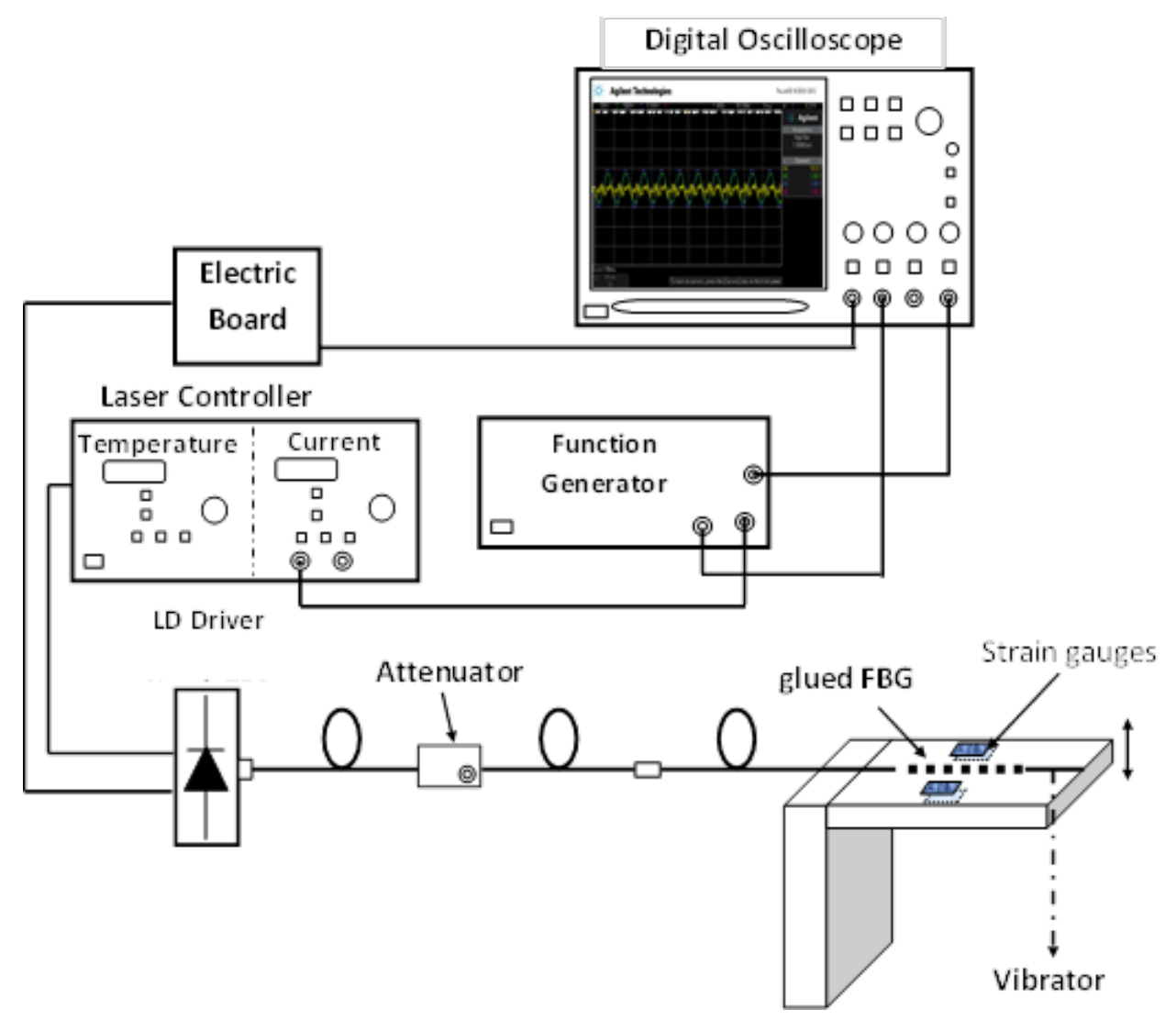

Fig. 1. Experimental scheme of optical feedback-based FBG strain sensor.

wave vibrator and the FBG was fixed onto the free part of the cantilever beam. The strain effects modified the FBG characteristics (the fluctuation elongation of the Bragg grating) are then translated into modification of the output characteristics of a single-mode DFB laser. This phenomenon occurs when the light emitted by the diode laser propagates through the fiber Bragg grating with a wavelength $\lambda_{B}$, a fraction of this light is reflected back into the active region of the laser (laser cavity) after reflection from the fiber Bragg grating, with condition that laser light wavelengths lie in the range of FBG spectrum ( $\lambda_{B} \pm \Delta \lambda_{B}$ with $\Delta \lambda_{B}$ is the FBG bandwidth). It consequently induces the interaction between re-injected electric field and stationary field inside the laser cavity. This interaction results in the effect of altering the power and frequency of laser emission. The longitudinal dynamic strain information has been directly detected from the obtained saw tooth-type optical self-mixing interference signals. The induced dynamic strain is calculated from singlemode FBG sensor by the detected fringe number of the obtained self-mixing signals recorded on the oscilloscope, given by [7]:

$$
\varepsilon_{z}=\frac{\Delta L}{L_{G}}=N \frac{\lambda_{B}}{2 n_{e f f} L_{G}}
$$


where $\lambda_{B}$ is FBG central wavelength, $n_{e f f}$ is the effective refractive index of silica fiber core $\left(n_{e f f}=1.468\right.$ for calculation in our work), $L_{G}$ is the length of the fiber comprising the FBG adhered to the plate (the gauge length) in experiment, and $N$ is fringe number of obtained selfmixing signals. The fringe number of signal can be obtained via oscilloscope. Schematic diagram of dynamic strain sensor using a DFB-FBG combination is shown in Fig. 1.

\section{II.2. Principle of temperature sensor using FBG}

The fractional FBG wavelength shifts for a temperature change $\Delta T$ and pressure $\Delta \mathrm{P}$ caused by the changes in grating spacing and effective index may be written as follows [8]:

$$
\frac{\Delta \lambda}{\lambda_{B}}=[\alpha+\xi] \Delta T+\left[-\frac{1-w v}{E}+\frac{n_{e f f}^{2}}{2 E}(1-2 v)\left(2 p_{12}+p_{11}\right)\right] \Delta P
$$

where: $\alpha=(1 / \Lambda)(\partial \Lambda / \partial T)$ is thermal expansion coefficient $\left(0.55 \times 10^{-6} \mathrm{~K}^{-1}\right.$ for silica), and $\xi=(1 / n)(\partial n / \partial T)$ is the thermo-optic coefficient, which is of $8.6 \times 10^{-6} \mathrm{~K}^{-1}, v$ is Poisson's ratio, $p_{11}$ and $p_{12}$ are components of strain-optic tensor, $E$ is modulus of elasticity for a germaniumdoped silica fiber. The schematic diagram of wavelength shift measurement using photodiode is shown in Fig. 2.

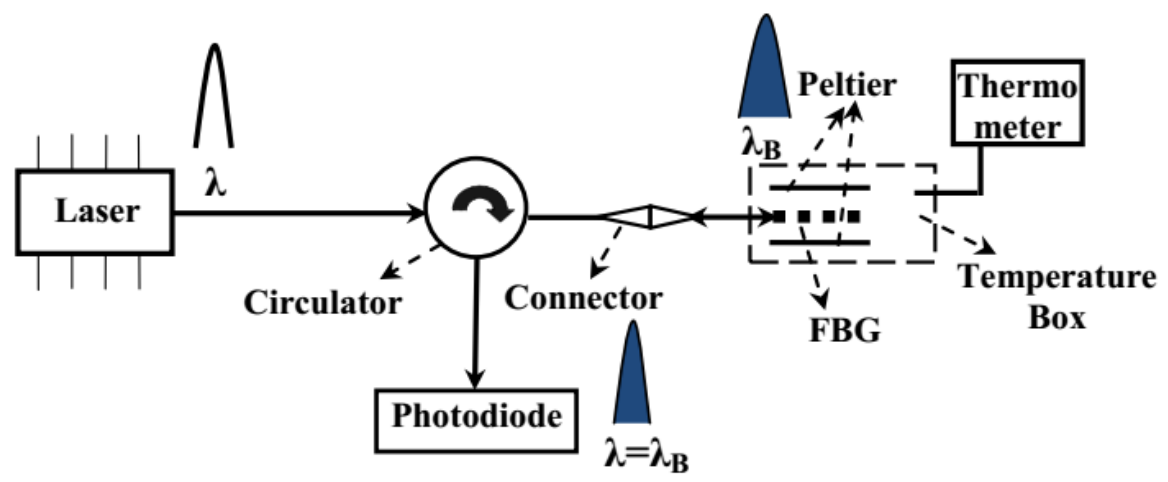

Fig. 2. Schematic diagram of wavelength shift measurement using photodiode.

We intend to compare the change of reflection Bragg wavelength, which shifts due to physical parameters such as temperature and/or pressure, and adjustment of emission wavelength of single-mode laser by controlling temperature of laser substrate. Fig. 2 shows emission wavelength of single-mode laser connect to circulator device and laser beam propagates to fiber Bragg grating, which has the FBG reflection wavelength equaled to laser wavelength at room temperature. The reflection light from FBG is collected by photodiode [9]. The opto-electrical intensity of photodiode current strongly depends on the ratio of the incident intensity and reflected intensity from FBG. The intensity of reflection light from FBG expressed by a modified Gaussian function as follows [10]:

$$
I_{\text {ref }}=R_{F B G} I_{\text {in }} \exp \left(-\Delta \lambda^{2} / \Delta \lambda_{0}^{2}\right),
$$

where $R_{F B G}$ is reflectivity of FBG; $I_{i n}$ is incoming light intensity; $\Delta \lambda_{0}$ is bandwidth of signal spectrum and $\Delta \lambda$ is spacing between Bragg wavelength center $\lambda_{B}$ and signal wavelength $\lambda$. The 
reflection intensity is maximal when the wavelength spacing between incoming and reflection is zero. In principle, we can control the emitted wavelength from laser by changing the temperature of laser substrate. If the wavelength shifts of lasing emission by temperature $\Delta \lambda_{L}$ are equal to FGB wavelength shift $\left(\Delta \lambda_{B}\right)$, so the photo-electrical intensity of photodiode becomes maximal again. That means we can measure the physical impact of environment by the adjustment of temperature of laser substrate without use of spectrometer.

\section{Principle of the e-FBG and fiber ring laser combined configuration}

Normal FBGs are intrinsically insensitive to the ambient refractive index. However, if the fiber cladding diameter is reduced along the grating region such that core mode interacts with the surrounding environment, the value of effective refractive index of the waveguide mode is directly affected by the external medium refractive index, leading to a shift in the reflected wavelength $\left(\lambda_{B}\right)$. For FBG bio-chemical sensor, the change of Bragg wavelength is due to change in refractive index induced by change in the chemical composition around the sensor. It is important for the optical mode to penetrate evanescently into the surrounding solution. When the optical fiber is etched to a point where the fundamental waveguide mode is affected, the modified propagation constant of the mode can be written as [11]:

$$
\beta=\beta_{0}+k \eta_{p}\left(n_{\text {ext }}-n_{c l}\right),
$$

where $\beta_{0}=(2 \pi / \lambda) n_{\text {eff }}$ is propagation constant of core mode under normal conditions, next is refractive index of external medium, $n_{c l}$ is refractive index of cladding, $k$ is wave vector, $\eta_{p}$ is the fraction of the total power of unperturbed fundamental mode that flows in the etched region and therefore lost to the external medium. The FBG etching process is associated with variation of Bragg wavelength of FBG and when etching process is stopped, $\eta_{p}$ becomes constant value, given by [12]: $\partial \lambda_{B}=2 \Lambda \eta_{p}\left(n_{e x t}-n_{c l}\right)$. In FBG bio-chemical sensor, the variation of the Bragg wavelength of FBG depends only on the variation of the refractive index of the external medium while the gratings pitch is practically unchanged.

For low-cost bio-chemical sensor, the configuration using dual fiber Bragg gratings (etchedFBG and reference-FBG) integrated in fiber ring laser structure is shown high sensitivity and effectively for determination of bio-chemical agents in liquid environment $[13,14]$. The sensing signal is demodulated the central wavelength shift of the e-FBG on the basis of the lasing power when lasing signal of the re-FBG is swept over the spectrum of the e-FBG by using the Peltier cooler that applies temperature on the re-FBG. The schematic of bio-chemical sensor system using dual FBGs is shown in Fig. 3.

In this configuration, the optical gain medium was an erbium-doped silica fiber with erbium concentration from $1500 \mathrm{ppm}$ to $4000 \mathrm{ppm}$ and with length of some meters (from $3 \mathrm{~m}$ to 15 $\mathrm{m})$. The optical pump was a $980 \mathrm{~nm}$-laser diode with adjusted output optical power up to 170 $\mathrm{mW}$ in single-mode emission (SDLO-2564-170). The pumped light was through a 980/1550 nm wavelength division multiplexer (WDM) to erbium-doped silica fiber for excitation of the erbium ions. The other end of the erbium-doped fiber was connected to a fiber-optic isolator in order to ensure that the lasing only occurs at one direction around the loop. Both FBGs, e-FBG and re-FBG function as a sensing element and filter, respectively, after connected to the ring cavity via two fiber-optic circulators. A light emitted from broadband amplified spontaneous emission of erbium ions enters the re-FBG through a circulator 1, and the reflection spectrum of re-FBG 
then propagates back through the circulator 1 again to the circulator 2, where the reflected signal is entered the e-FBG then filtered and reflected again into loop-cavity. The laser emission is directed out by a fiber-optic coupler 10/90 in order to extract $10 \%$ of the light from the cavity to be detected by a photo-detector, and $90 \%$ of the light comes back to the laser cavity loop [14].

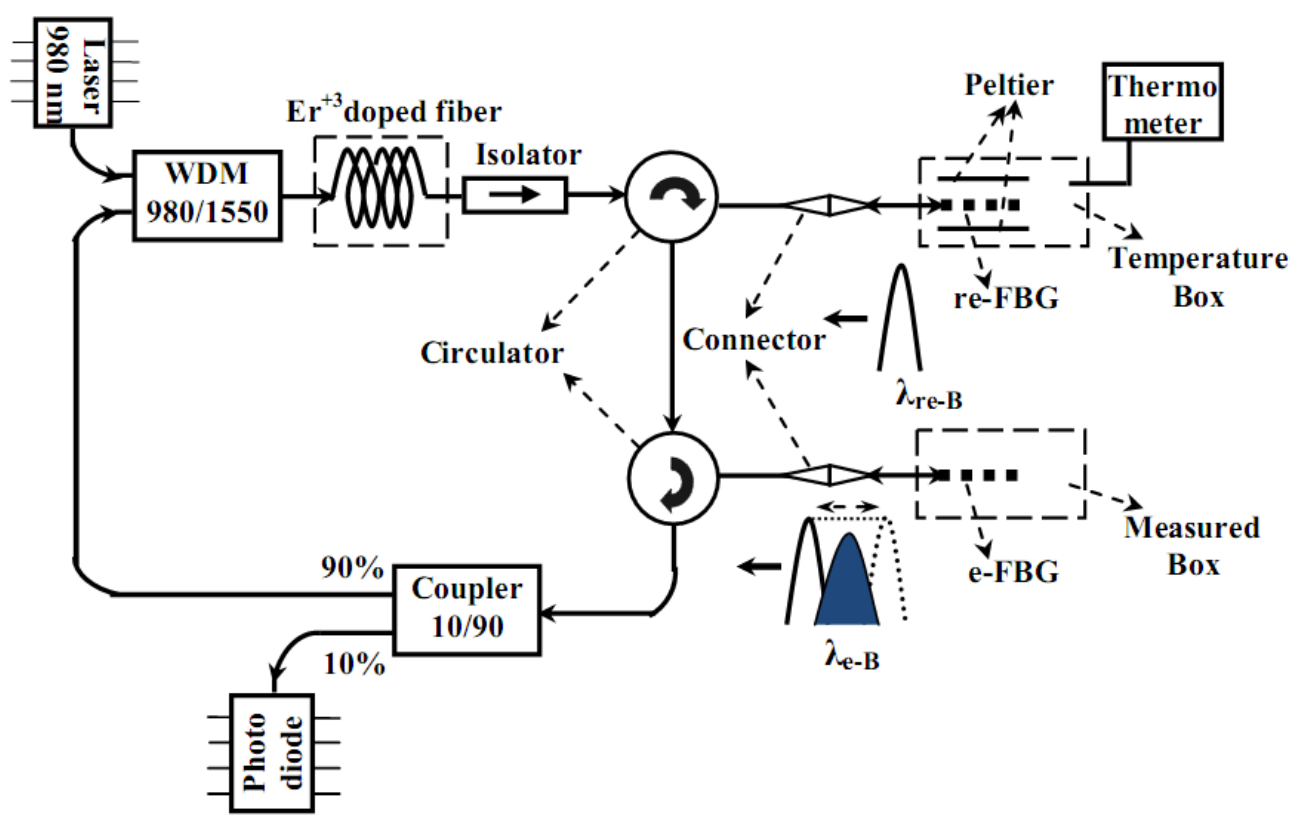

Fig. 3. Schematic diagram of the low-cost bio-chemical sensor using dual fiber Bragg gratings (e-FBG and re-FBG) integrated in fiber ring laser configuration [13, 14].

\section{EXPERIMENT AND RESULTS}

\section{IV.1. Results of dynamic strain sensor}

As shown in Fig. 1, the strain sensor configuration consists of a fiber pigtailed DFB-type laser diode package incorporating a photodiode without optical isolation emitting at $1310 \mathrm{~nm}$, coupled to a fiber Bragg grating. The FBG is mounted and glued with the length LG of $45 \mathrm{~mm}$ onto the cantilever-beam using epoxy (a thin aluminum plate with elastic modulus $\mathrm{E}=70 \mathrm{GPa}$ ). This plate is then subjected to sinusoidal vibration inducing longitudinal dynamic strain on the Bragg grating. The vibrator is made by the SF-9324 Variable Frequency Mechanical Wave Drive (PASCO Scientific), it is a strong and long-throw speaker vibrated with any frequency of waveform generator from $0.1 \mathrm{~Hz}$ to $5 \mathrm{kHz}$ and amplitudes up to $7 \mathrm{~mm}$-p at the low end of the frequency range, which is applied to the below side free end of the cantilever beam and driven by a sinusoidal function generator with frequency $20 \mathrm{~Hz}$. On cantilever-beam, the reference Gauge sensor formed by four the electrical strain gauges (interrogated by a full Wheatstone bridge configuration) is mounted as close as possible to the FBG with glued two into the upper surface and the other two on the underside of the thin aluminum plate, and to measure the dynamic strain directly by two techniques such as the reference Gauge sensor and the single-mode FBG dynamic strain sensor 
in the same experimental conditions. For measurement of longitudinal dynamic strain we carry out at room temperature $\mathrm{TR}=20^{\circ} \mathrm{C}$. The emitting wavelength, operating current and temperature of the DFB- laser (LD1310) are $1310.02 \mathrm{~nm}, 34.5 \mathrm{~mA}$ and $20^{\circ} \mathrm{C}$, respectively. The FBG central wavelength is $1310.04 \mathrm{~nm}$ with FWHM spectrum width of $\Delta \lambda=0.14 \mathrm{~nm}$, and the FBG reflectivity RFBG is $19 \%$ from incoming laser power. When lasing wavelength lies on FBG spectrum range, the emission light is reflected back into the active cavity of laser diode. If cantilever-beam is vibrated by a mechanical wave vibrator excited by a sinusoidal function generator with different excitation amplitudes at a frequency of $20 \mathrm{~Hz}$ corresponding to the different longitudinal dynamic strain, and applied to the FBG inducing elongation or compression of the Bragg grating. Therefore, the modulation of external cavity length is produced, laser operation is altered, causing self-mixing interferometry phenomenon and to be detected by the photo-detector (PD) in the laser diode package. The fluctuations emitted power of optical output laser as a function of time is then amplified by an external electronic circuit and transferred to a digital oscilloscope for display [15]. The induced dynamic strain according to the excited cantilever-beam by a mechanical wave vibrator driven a sinusoidal function generator with different excitation amplitudes (corresponding to the various output voltage of the Wheatstone bridge from $0.24 \mathrm{~V}$ to $1.15 \mathrm{~V}$ with change step of $0.02 \mathrm{~V}$ ) at a frequency $20 \mathrm{~Hz}$ is applied to the Bragg grating and the induced dynamic strains are measured by the reference Gauges sensor. The obtained self-mixing signals from FBG strain sensor subject to dynamic strain are displayed on the oscilloscope.

Table 1. The measured dynamic strain of reference Gauges sensor and single-mode FBG sensor by fringe counting method for the applied dynamic strain by a mechanical wave vibrator driven a sinusoidal function generator with various excitation amplitudes at frequency $20 \mathrm{~Hz}$.

\begin{tabular}{|l|c|c|c|c|}
\hline$V_{\text {Bridge }}(\mathrm{V})$ & $\begin{array}{c}\text { Gauges sensor } \\
\text { strain }(\mu \varepsilon)\end{array}$ & $\begin{array}{c}\text { Fringes } \\
\text { number } N\end{array}$ & $\begin{array}{c}\text { Single-mode FBG } \\
\text { sensor strain }(\mu \varepsilon)\end{array}$ & $\varepsilon_{F B G}-\varepsilon_{G}$ \\
\hline 0 & 0 & 0 & 0 & 0 \\
\hline 0.24 & 25.0836 & 3 & 26.5545 & 1.4708 \\
\hline 0.38 & 39.7157 & 5 & 44.2574 & 4.5417 \\
\hline 0.52 & 54.3478 & 6 & 53.1089 & 1.2389 \\
\hline 0.64 & 66.8896 & 8 & 70.8119 & 3.9223 \\
\hline 0.76 & 79.4314 & 9 & 79.6634 & 0.2319 \\
\hline 0.86 & 89.8829 & 11 & 97.3664 & 7.4834 \\
\hline 0.98 & 102.4247 & 12 & 106.2178 & 3.7931 \\
\hline 1.07 & 111.8311 & 13 & 115.0693 & 3.2382 \\
\hline 1.15 & 120.1923 & 14 & 123.9208 & 3.7285 \\
\hline
\end{tabular}

Table 1 summarizes the values of induced dynamic strain obtained from the reference strain gauge sensor and single-mode FBG strain sensor based on the output voltage of the Wheatstone 
bridge and counting the fringes number of the obtained self-mixing signals. The error of singlemode FBG sensor with Gauges sensor is less than $8 \%$. The induced dynamic strain results measured by the single-mode FBG strain sensor and reference Gauges strain sensor are plotted in Fig. 4, in order to confirming the feasibility of the demodulated technique of the FBG sensor by the principle of optical feedback interferometry.

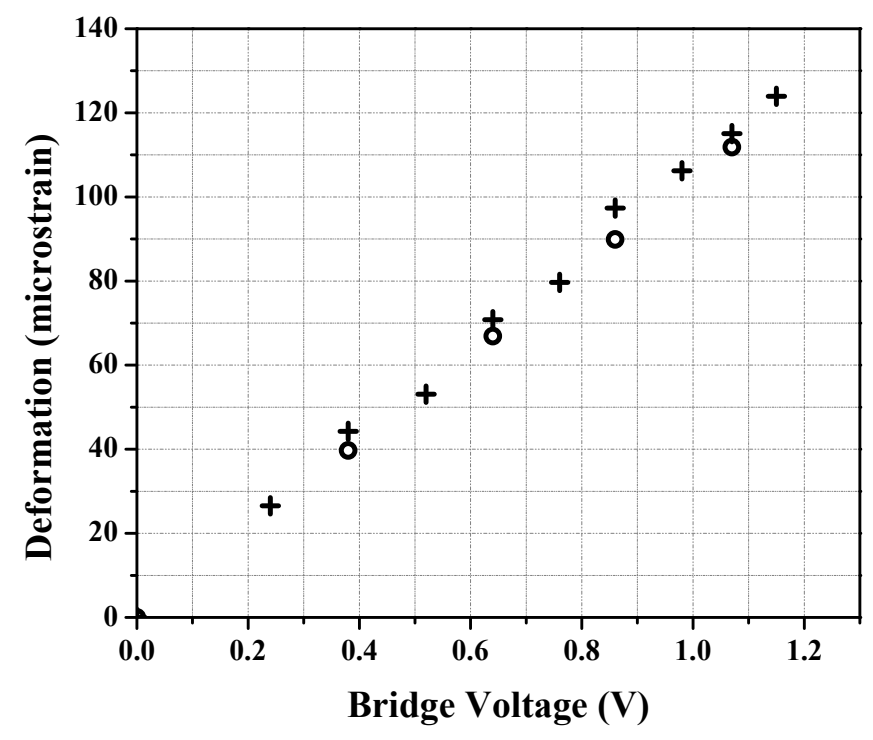

Fig. 4. Comparison the measured dynamic strain results of single-mode FBG sensor (cross points) and reference Gauges sensor (cycle points).

The resolution obtained from this FBG strain sensor was about $8 \mu \varepsilon$ which corresponded to a half-fringe $\frac{\lambda_{B}}{2 n_{e f f} L_{G}}$, and this could be further be improved by adopting a better signal processing scheme instead of the current fringe counting technique used in experiment.

\section{IV.2. Results of temperature sensors}

For testing the characteristics of FBG depended on the ambient temperature in the DFB-laser diode and FBG combined configuration, we use thermal box which can control the temperature from $278 \mathrm{~K}$ to $373 \mathrm{~K}$. The FBG can be recoated by different thermal-sensitive materials such as Teflon, polymer and metals [3] and embedded on the Copper $(\mathrm{Cu})$ substrate. We should choice the embedded FBG with polymer (silicone) which has a reflection wavelength at room temperature matching with the emission wavelength of DFB-laser diode. In this experiment, we used laser driver with feedback control for keeping the constant value of output laser power at changed temperature that is different with previous work in [16]. This technique is shown high accuracy of signal intensity measurement using photodiode. The dependence of the siliconeembedded FBG wavelength shift upon the ambient temperature, which is shown in Fig. 5a, is linear in the range of $278 \mathrm{~K}-373 \mathrm{~K}$. The silicone-embedded Bragg wavelength shift is approximately $3.0 \mathrm{~nm}$ and the average temperature sensitivity of the FBG corresponds to $27.2 \mathrm{pm} . \mathrm{K}^{-1}$. 

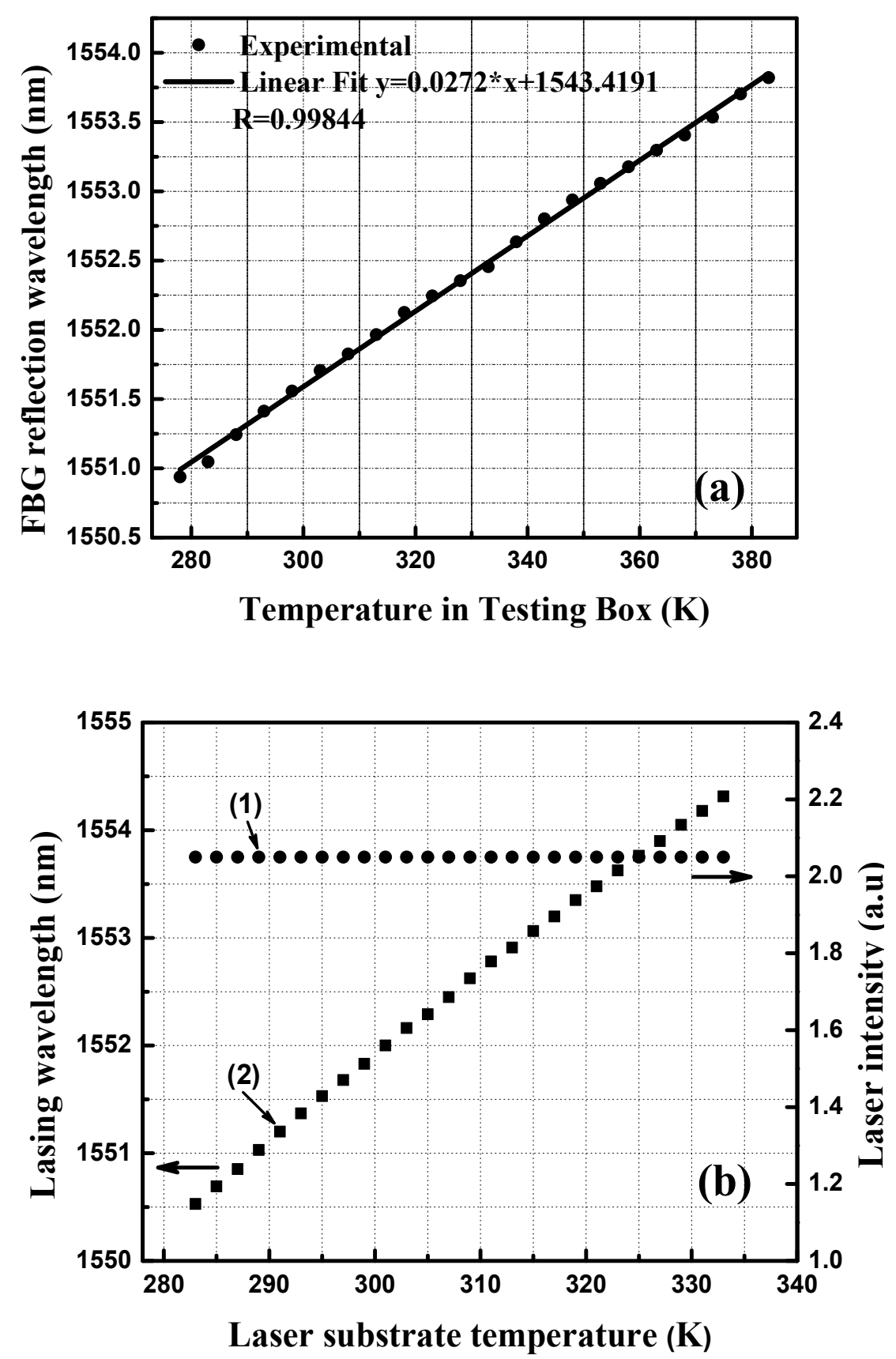

Fig. 5. Dependence of FBG-wavelength shift on the ambient temperature (a), and lasing intensity (circle points) with emission wavelengths (square points) of DFB-laser diode versus the laser substrate temperature (b). 
We use single-mode DFB-laser (2A0469) made by Photonics company with the spectral line-width and maximal output power of emission are $0.2 \mathrm{~nm}$ and $5 \mathrm{~mW}$, respectively. For testing tunable of lasing wavelength depended on the laser substrate temperature we use Peltier cooler which can adjust exactly the temperature $(0.1 \mathrm{~K})$ in the range of $283 \mathrm{~K}-333 \mathrm{~K}$. The dependence of lasing wavelength shift and lasing intensity of laser diode upon substrate temperature have been shown in Fig. 5b. The dependence of emitted wavelength shift upon the altering temperature is linear and the temperature sensitivity of laser emitted wavelength is $77.5 \mathrm{pm} . \mathrm{K}^{-1}$.

The wavelength shift of FBG sensor could be determined without using spectrometer, which is the way combination of FBG wavelength shift in the temperature range of $278 \mathrm{~K}-$ $373 \mathrm{~K}$ and the temperature of laser substrate in the range of $283 \mathrm{~K}-333 \mathrm{~K}$ with the photoelectrical maximal intensity of photodiode. The accuracy of measured photoelectrical signal is $\pm 0.02 \mathrm{~V}$. The photoelectrical maximal intensity of photodiode is $2.05 \pm 0.02 \mathrm{~V}$, when the laser wavelength and FBG reflection wavelength are coincided. It is remarkable that the FBG wavelength shift due to physical impacts of ambient can be measured by the change of laser substrate temperature without use of spectrometer, if we have a data base of the FBG wavelength shift due to the physical parameters and lasing wavelength shift due to the temperature change.

\section{IV.3. Results of bio-chemical sensors using e-FBG combined in the ring cavity fiber laser}

In experiment of the ring-cavity fiber laser combined with two FBGs, we used the re-FBG as the same characteristics as used in [14] (the Bragg wavelength at room temperature was in the range of $1530 \div 1550 \mathrm{~nm}$ and a reflectivity was $75-90 \%$; the full-width-half-maximum (FWHM) bandwidth of $0.15 \div 0.30 \mathrm{~nm}$ and FBG length of $15 \mathrm{~mm}$ ). But the e-FBG fabricated by wet chemical etching only half part of fiber cladding of the FBG region in hydrofluoric acid (HF) solution for creating D-form of FBG-fiber to increase the interaction of the propagating optical field in the FBG with the surrounding medium, which is different form in comparison with e-FBG presented in previous works $[14,17]$. In the e-FBG and fiber ring laser combined configuration, the reflected light from e-FBG can be used to analyze the interaction between the evanescent wave and the surrounding medium by measuring wavelength shift of lasing emission. Fig. 6a shows the spectra of reflection light from e-FBG and of lasing emission from ring fiber laser used the same e-FBG as reflector in solvent solution (Acetone of $99.9 \% \mathrm{v} / \mathrm{v}$ ). The optical signal-to-noise ratio (OSNR) and spectral line-width of reflection light from e-FBG are $13.5 \mathrm{~dB}$ and $0.24 \mathrm{~nm}$, but OSNR and spectral line-width of lasing light from ring fiber laser is of $39.4 \mathrm{~dB}$ and $0.02 \mathrm{~nm}$, respectively. The OSNR of the sensor depends on how accurately and precisely the sensor can detect the resonant wavelength shift of the sensing element [18]. Therefore, the fiber laser configuration using e-FBG sensing element sensor system has strongly enhanced detection accuracy of the wavelength peak measurement. This measuring configuration is demodulated the central wavelength shift of the e-FBG on the basis of the lasing power when lasing signal of the re-FBG is swept over the spectrum of the e-FBG by using the Peltier element that applies temperature on the re-FBG. At first, we should choice the re-FBG with reflection wavelength at room temperature equaled to the reflection wavelength of e-FBG in order to easy scanning the re-FBG signal over the spectrum of the e-FBG. The data base of wavelength shift of re-FBG in the temperature range of $283 \mathrm{~K}-343 \mathrm{~K}$ is shown in Fig. $6 \mathrm{~b}$. 


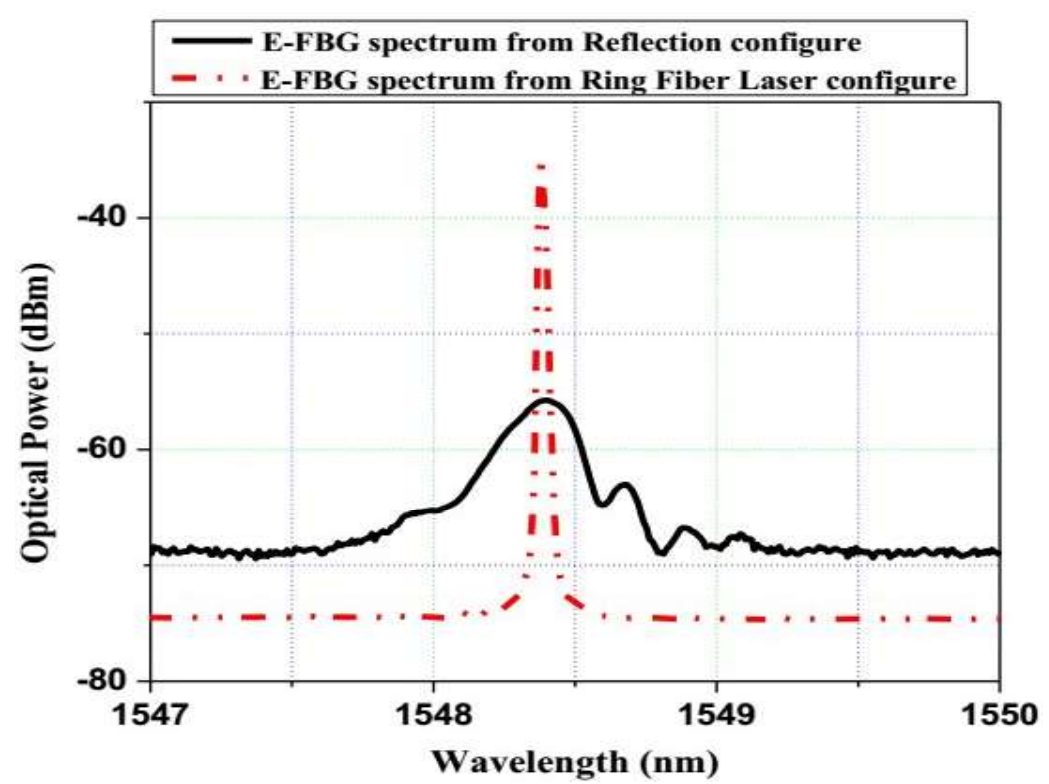

(a)

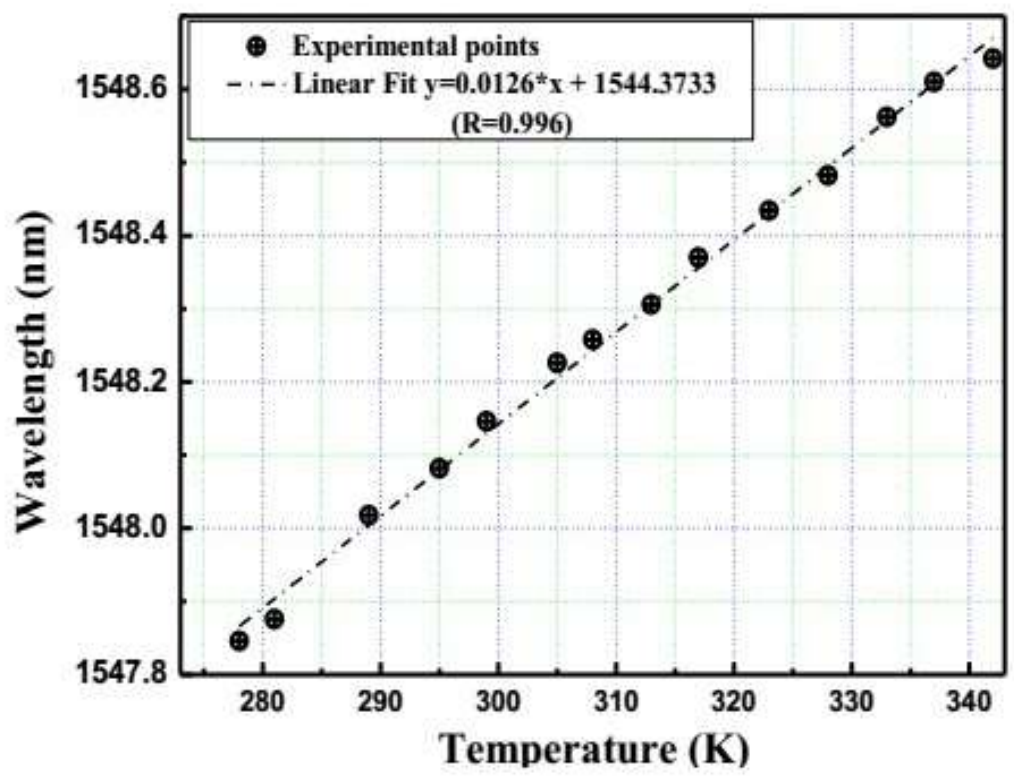

(b)

Fig. 6. Spectra of reflection light from e-FBG and of ring fiber laser using the same e-FBG in solvent solution (a), and dependence of re-FBG wavelength shift on temperature (b). 
It is observed that the dependence of the re-FBG wavelength shift on temperature is linear and the temperature sensitivity of the re-FBG corresponds to $12.6 \mathrm{pm} . \mathrm{K}^{-1}$ in temperature range of $278 \mathrm{~K}-343 \mathrm{~K}$. This measurement demodulates the central wavelength shift of the sensing e-FBG on the basis of monitoring the power change of laser signal, which is caused by the overlap between the reflection spectrum of the reference-FBG and the e-FBG (Fig. 7a).

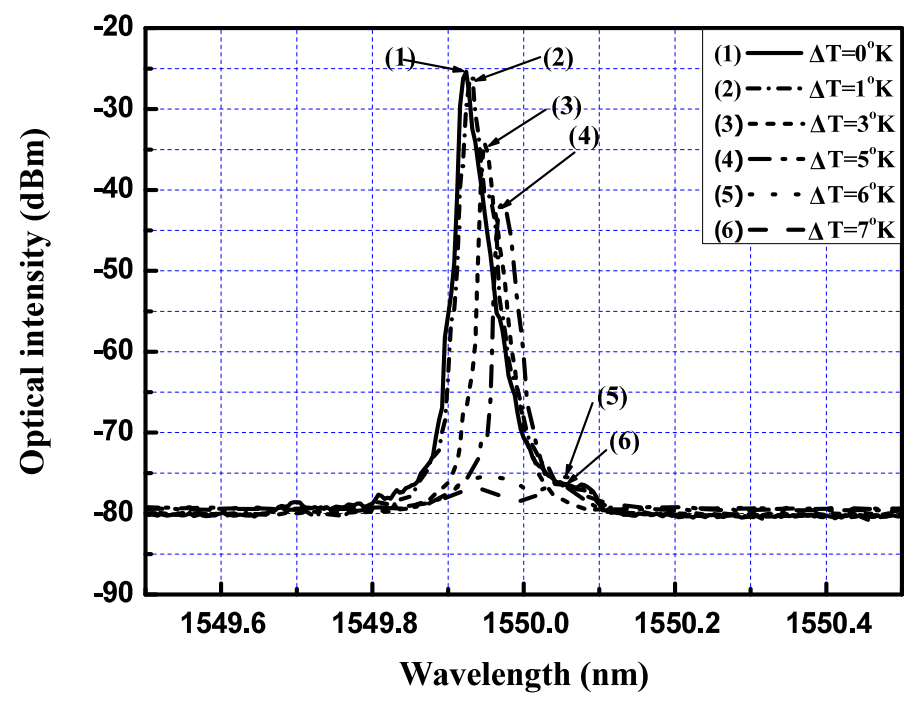

(a)

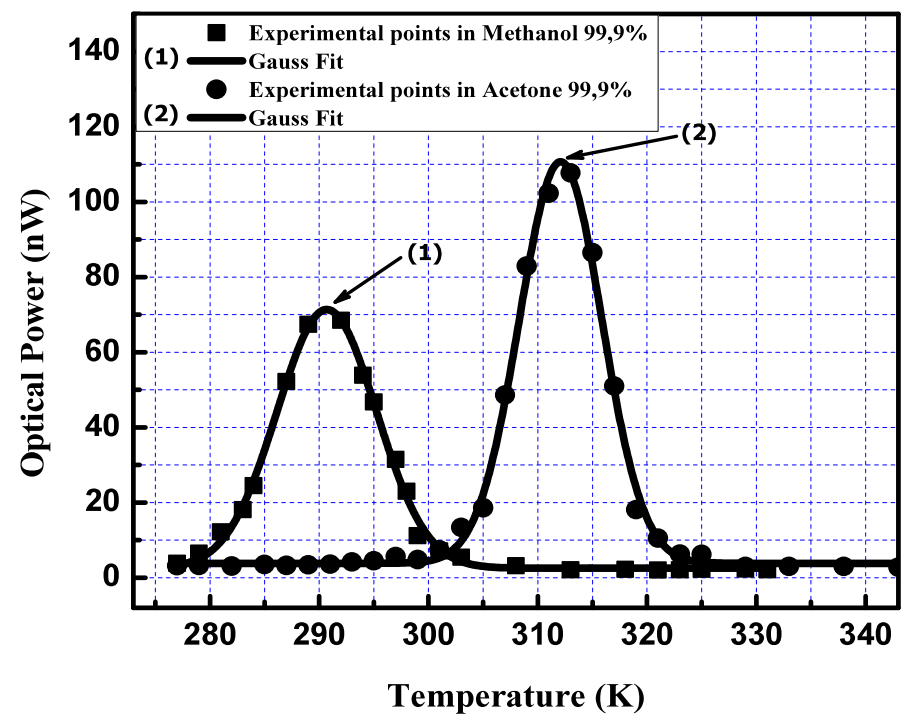

(b)

Fig. 7. Variation of lasing signal in intensity and wavelength as scanning the reflected signal of re-FBG by different temperature between two FBGs (a), and curves of laser power change versus re-FBG temperature for Methanol and Acetone solution of $99.9 \%$ (b). 
Due to the effect of the index refractive of analytic environment, the central wavelength of the e-FBG shifts and to be determined by scanning the reflected signal of re-FBG over the spectrum of the e-FBG through using the Peltier element applied temperature. When the reflected signal of re-FBG is coincided exactly with the spectrum of the e-FBG $\left(\lambda_{r e-B}=\lambda_{e-B}\right)$, the measured optical power lasing signal is maximum (Fig. 7b).
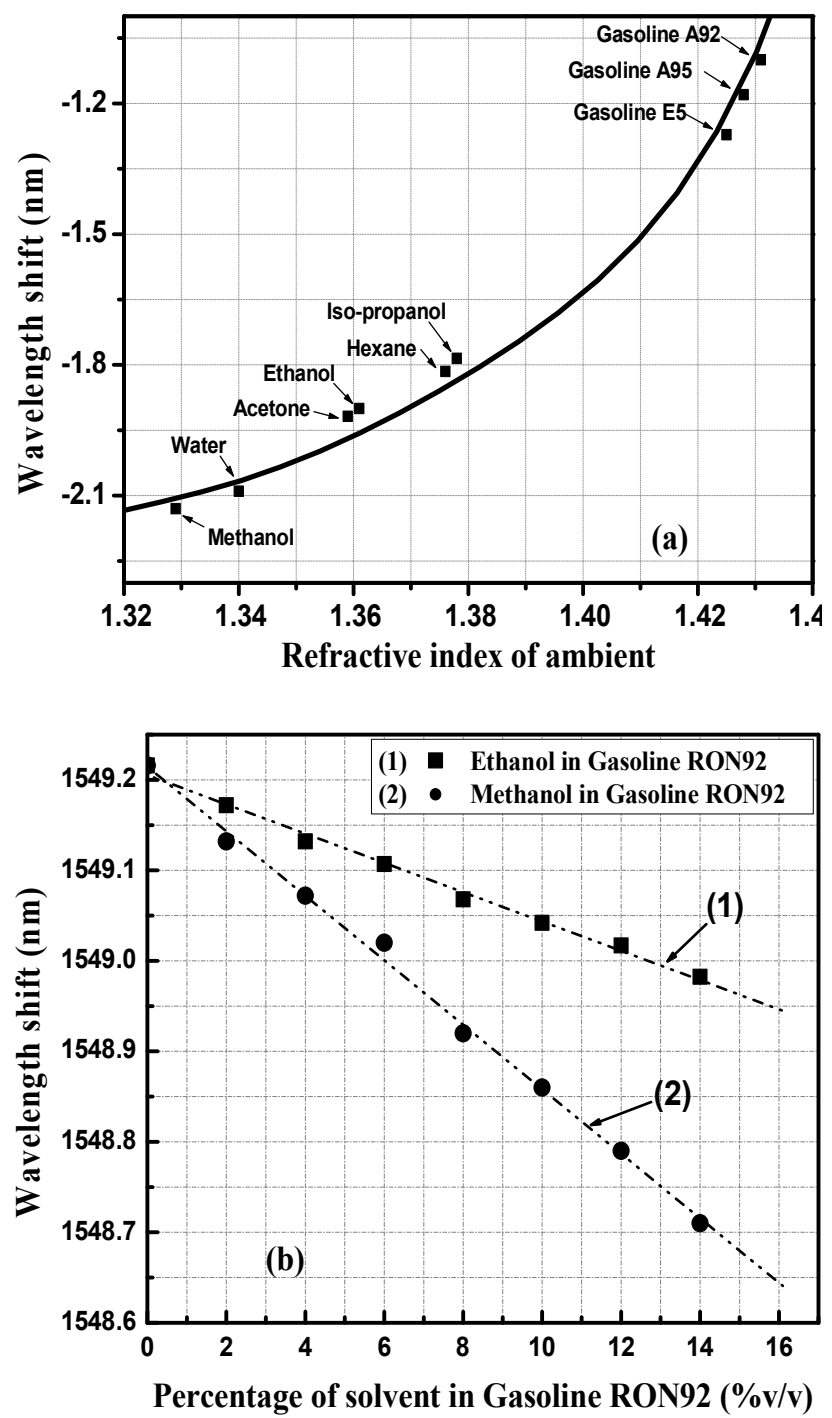

Fig. 8. The determined e-FBG wavelength shift for different organic solvents and gasolines with different refractive indices (a), and different of Ethanol and Methanol concentration in gasoline RON92.

Fig. 8a shows the determined e-Bragg resonant wavelength shift as a function of the refractive index of the surrounding medium at room temperature. The Bragg resonant wavelength 
increases with the increment of the refractive index. In order to demonstrate the determination of ethanol concentration in bio-gasoline, we have studied the e-FBG sensor response for commercial gasoline RON92 blended with ethanol and/or methanol in the concentration range of $0 \%-14 \% \mathrm{v} / \mathrm{v}$. The experimental results are depicted in Fig. 8b.

From Fig. 8b, the calculated slope of linearly fitted data can be used as the effective sensitivity of the FBG sensor. It can be deduced that the sensitivity of the achieved e-FBG sensor is about $45 \mathrm{~nm} / \mathrm{RIU}$ in the refractive index range of 1.41-1.43, which is corresponding to blend ethanol concentration from $0 \%$ to $14 \% \mathrm{v} / \mathrm{v}$ in commercial gasoline RON92. Therefore, assuming that the detectable spectral resolution is $10 \mathrm{pm}$, the optical sensor can measure solvent concentration blended into gasoline with accuracy of $0.2 \% \mathrm{v} / \mathrm{v}$ and $0.15 \% \mathrm{v} / \mathrm{v}$ for ethanol and methanol, respectively. The minimum detectable refractive index resolution of sensing measurement is of $2 \times 10^{-4}$. In addition, the slopes of wavelength shift curves from ethanol and methanol mixtures are different, that we can distinguish the organic solvent additive in gasoline by the data-base of a concrete mixture.

\section{CONCLUSION}

We overview three configurations of FBG sensors for measurement of dynamic strain, surround temperature and organic compounds in the liquid environment without use of spectrometer. In the single-mode FBG strain sensor, the dynamic strain can be determinated by fringe number of self-mixing modulation of lasing emission. The resolution of FBG strain sensor is about $8 \mu \varepsilon$, which is suitable for dynamic strain sensor for hazardous environment. The second kind of FBG sensor can detect wavelength shift by change of the laser substrate temperature for the matching the wavelength of single-mode laser emission and silicone-embedded FBG reflection wavelength, when the FBG influenced by physical parameters such as temperature and/or pressure from environment. This sensor has low cost and can be applied to different measurements in the real environment. The last type of FBG sensor using etched-FBG as reflector in the ring-cavity fiber laser is suitable for detection of different types of organic solvents in liquid environment. Moreover, the FBG sensor system shows many advantages including a high resolution for wavelength shift identification, high OSNR and narrow band-width that enhances accuracy and precision of wavelength peak measurement and improves capability for remote sensing application. In order to confirming the feasibility of determination of mixture gasoline, the e-FBG sensor has distinguished different types of gasoline with different octane numbers (RON 92 and RON95), and ethanol or methanol concentration in gasoline blended specifically in the low concentration range of $0 \%-14 \% \mathrm{v} / \mathrm{v}$ with mixture concentration accuracy of $0.2 \% \mathrm{v} / \mathrm{v}$ and $0.15 \% \mathrm{v} / \mathrm{v}$ for ethanol and methanol, respectively. These results point to the prospect of using the low-cost optical sensor for application in the civil engineering, agriculture, industrial fluids, and the food industry.

\section{ACKNOWLEDGMENTS}

This work is financially supported by the National Foundation for Science and Technology Development of Vietnam (NAFOSTED) under grant No. 103.03-2015.23. This work had used the apparatus of National Key Laboratory for Electronic Materials and Devices in Institute of Materials Science. 


\section{REFERENCES}

[1] B.N. Shivananju, S. Yamdagni, R. Fazuldeen, A. K. Sarin Kumar, G. M. Hegde, M. M. Varma, S. Asokan, Rev. Sci. Instrum. 84 (2013) 065002.

[2] N. Cennamo, D. Massarotti, L. Conte, L. Zeni, Sensors 11 (2011) 11752.

[3] V. H. Pham, T. B. Pham, T. T. A. Pham, X. V. Ha, T. T. H. Chu, T. T. Nguyen, Sens. Actuators A: Physical 141 (2008) 334.

[4] W. Liang, Y. Huang, Y. Xu, R.K. Lee, A. Yariv, Appl. Phys. Lett. 86 (2005) 151.

[5] G. Wild and S. Hinckley, IEEE Sensors J. 8 (2008) 1184.

[6] Q. Wu and Y. Okabe, Opt. Express. 20 (2012) 28353.

[7] D. Tosi, M. Olivero and G. Perrone, Electron. Lett. 44 (2008) 405.

[8] K. O. Hill, G. Meltz, J. Light. Technol. 15 (1997) 1263.

[9] V. H. Pham, H. Bui, T. V. Nguyen, T. A. Nguyen, T. B. Pham, T. C. Tran, H. T. Le, V. D. Pham, Vietnamese Patent No.17177, 2017

[10] G. M. H. Flockhart, R. R. J. Maier, J. S. Barton, W. N. MacPherson, J. D. C. Jones, K. E. Chisholm, L. Zhang, I. Bennion, I. Read and P. D. Foote, Appl. Opt. 43 (2004) 2744.

[11] A. W. Snyder and J. D. Love, Optical Waveguide Theory, Chapmann \& Hall, London, 1983.

[12] D. A. Pereira, O. Frazzo and J. L. Santos, Opt. Engineering 43 (2004) 299.

[13] T. B. Pham, V. H. Pham, H. Bui, T. H. Phung, V. T. Nguyen, H. T. Le, V. A. Nguyen, Q. M. Ngo, V. D. Pham, Submitted Vietnamese patent No.50711 Industrial Property Official Gazette vol. A, No.329 92, 2017

[14] H. Bui, T. B. Pham, V. A. Nguyen, V. D. Pham, T. C. Do, T.V. Nguyen, T. H. C. Hoang, H. T. Le, and V. H. Pham, Meas. Sci. Technol. 29 (2018) 055105

[15] T. B. Pham, H. C. Seat, O. Bernal. M. Suleiman, IEEE Sensors J. 11 (2012) 1341.

[16] Nguyen The Anh, Pham Thanh Son, Nguyen Thuy Van, Hoang Thi Hong Cam, Ngo Quang Minh, Bui Huy, and Pham Van Hoi, Comm. Phy. 23 (2013) 67.

[17] T.B. Pham, H. Bui, H.T. Le, V.H. Pham, Sensors 17 (2017) 7, doi:10.3390/s17010007

[18] M. S. Ferreira, J. Bierlich, M. Berker, K. Schuster, J. L. Santos and O. Frazao, Fiber 2 (2014) 142. 